2. Висков, А. Н. Влияние температуры струи, направленной перпендикулярно набегающему потоку, на подъемную силу треугольного крыла [Текст] / А. Н. Висков, А. Ф. Ражин, С. И. Руденко // Ученые записки ЦАГИ. - 1971. - Том II, № 2. - С. 98-100.

3. Ву, Т. Ч. Исследование влияния теплообмена на подъемную силу модели прямоугольного крыла при дозвуковых скоростях [Текст] / Т. Ч. Ву, В. В. Вышинский // Труды МФТИ. - Сер. Аэрогидромеханика. - 2013. - Том 5. - № 2. - С. 88-93.

4. Ву, Т. Ч. Исследование влияния теплообмена на аэродинамические характеристики модели прямоугольного крыла при дозвуковых скоростях [Текст] / Т. Ч.Ву, В. В. Вышинский, Н. Т. Данг // Труды МФТИ. - Сер. Аэрогидромеханика. - 2012. - Том 4. - № 2. - C. $148-153$.

5. Вышинский, В. В. Аэродинамические характеристики профиля крыла с учетом теплообмена с потоком вязкого, сжимаемого газа при дозвуковых скоростях [Текст] / В. В. Вышинский, А. С. Петров, Ву Тхань Чунг // Научный вестник МГТУ ГА. Сер. Аэромеханика и прочность. - 2010. - № 151. - С. 7-11.

6. Вышинский, В. В. Аэродинамические характеристики профиля крыла с учетом теплообмена с потоком вязкого, сжимаемого газа при дозвуковых скоростях [Текст] / В. В. Вышинский, А. С. Петров, Т. Ч. Ву // Труды XV Международного симпозиума «Методы дискретных особенностей в задачах математической физики». - Харьков-Херсон. - 2011. C. $115-118$.

7. Исаченко, В. П. Теплопередача [Текст] /В. П. Исаченко, В. А. Осипова, А. С. Сукомел. - М.: Энергия, 1975. - 488 с.

8. Кравец, Е. В. Гидродинамика течения между прямоугольными призмами, расположенными тандемом над экраном [Текст]: Диссерт. на соиск. ученой степени к. ф.м. н. по спец. 01.02 .05 - Механика жидкости, газа и плазмы / Е. В. Кравец. - Днепропетровск, 2009. $-229 \mathrm{c}$.

9. Петров, А. С. Теория аэродинамических сил при дозвуковых скоростях: учебное пособие [Текст] / А.С. Петров. - М.: МФТИ, 2007. - 236 с.

10. Kravets, E. V. Influence of Turbulence Model on Exactness Calculation of Wing Aerodynamics in Subsonic Stream [Текст] / E. V. Kravets // Вісник Дніпропетровського університету. Сер. Механіка. - 2018. - Вип. 22. - № 5. - Т. 26. - С. 96-102.

Надійшла до редколегї 01.03.2019

УДК 532.3

О. Г. Гоман, Т. М. Никулина

Днепровский национальный университет имени Олеся Гончара

\title{
УДАРНОЕ ВЗАИМОДЕЙСТВИЕ ТЕЛА В ВИДЕ КРУГОВОГО СЕГМЕНТА С ЖИДКОСТЬЮ С ОБРАЗОВАНИЕМ ЗОНЫ ОТРЫВА
}

\footnotetext{
В работе в плоской постановке рассматривается задача об ударе гладкого криволинейного тела, предварительно погруженного в жидкость, занимающую безграничное полупространство. Жидкость считается несжимаемой, а погруженная в жидкость часть тела имеет форму кругового сегмента. Предполагается, что в некоторый момент времени происходит нецентральный удар, в результате которого тело мгновенно получает горизонтальную $U$ и вертикальную $V$ скорость движения, а также угловую скорость вращения $\omega$ вокруг оси, перпендикулярной плоскости, в которой рассматривается течение.
}

(C) Гоман О. Г., Никулина Т. М., 2019 
Предполагается также, что при определенной комбинации кинематических и геометрических параметров погруженной части тела в виде сегмента, жидкость может мгновенно оторваться от поверхности тела и образовать дополнительный участок свободной поверхности. Сложность задачи состоит в том, что положение отрывной зоны (координата крайней ее точки) заранее неизвестно; оно зависит от комбинации кинематических и геометрических параметров. Появление зоны отрыва существенно осложняет исходную гидродинамическую задачу, поскольку поле скоростей жидкости зависит от положения зоны отрыва, а геометрические параметры этой зоны, в свою очередь, зависят от комбинации кинематических параметров.

В работе для определения положения зоны отрыва (крайней ее точки) использован так называемый принцип Огазо, выражающий вариационный принцип, который состоит в том, что реализуемое в действительности отрывное течение жидкости обеспечивает экстремальное значение потенциала среди других возможных решений смешанной ударной задачи гидромеханики. Данный принцип позволяет отсеять все те возможные математические решения, которые допускают наличие на поверхности контакта тела с жидкостью отрицательных импульсов, что противоречит физической сущности гидродинамических явлений.

Общее решение задачи об определении поля скоростей и импульсов в жидкости в момент, следующий непосредственно за ударом, с заранее произвольным параметром, характеризующим величину участка отрыва, в работе получено при помощи конформного отображения области, занятой жидкостью (полуплоскости с вырезанным сегментом) на вспомогательную полуплоскость с последующим сведением исходной задачи к задаче Келдыша-Седова для этой полуплоскости.

Весьма существенным является тот момент, что применение принципа Огазо приводит к трансцендентному уравнению для определения параметра q, определяющего положение крайней точки зоны отрыва, содержащему сингулярные интегралы, которые следует понимать в смысле конечной части по Адамару.

Численная процедура, основанная на использовании метода Адамара-Манглера, позволила определить значение параметра q как функцию кинематических параметров и геометрического параметра $\alpha$, характеризующего указанный сегмент.

После того как определен параметр q, определяющий положение зоны отрыва, определение потенциала сводится к вычислению некоторого интеграла, понимаемого в смысле Коши. В работе представлены результаты расчета импульсивного давления по поверхности сегмента с учетом существования участка отрыва потока.

Ключевые слова: несжимаемая жидкость, ударная задача для жидкости, отрыв жидкости от твердой поверхности, задача Келдыша-Седова.

У роботі в плоскій постановці розглянуто задачу про удар гладкого криволінійного тіла, попередньо зануреного в рідину, що займає безмежний напівпростір. Рідина вважається нестисливою, а занурена в рідину частина тіла має форму кругового сегмента. Вважається, що в певний момент часу відбувається нецентральних удар, в результаті якого тіло миттєво отримує горизонтальну $U$ i вертикальну $V$ швидкість руху, а також кутову швидкість обертання $\omega$ навколо осі, перпендикулярної площині, в якій розглядається течія.

Вважасться також, що при певній комбінації кінематичних і геометричних параметрів зануреної частини тіла у вигляді сегмента, рідина може миттсво відірватися від поверхні тіла і утворити додаткову ділянку вільної поверхні. Складність задачі полягас в тому, що положення відривної зони (координата крайньої ії точки) заздалегідь невідомо; воно залежить від комбінації кінематичних і геометричних параметрів. Виникнення зони відриву істотно ускладнюс вихідну гідродинамічну задачу, оскільки поле швидкостей рідини залежить від положення зони відриву, а геометричні параметри цієї зони, у свою чергу, залежать від комбінації кінематичних параметрів.

У роботі для визначення положення зони відриву (крайньої її точки) було використано так званий принцип Огазо, що виражає варіаційний принцип, який полягає в тому, що відривна течія рідини забезпечус екстремальне значення потенціалу серед усіх інших можливих рішень змішаної ударної завдання гідромеханіки. Даний принцип дозволяє відсіяти всі ті можливі математичні рішення, які допускають наявність на поверхні контакту тіла 3 рідиною від'ємних імпульсів, що суперечить фізичної сутності гідродинамічних явищ.

Загальний розв'язок задачі про визначення поля швидкостей і імпульсів в рідині в момент, наступний безпосередньо за ударом, із заздалегідь довільним параметром, що характеризує величину зони відриву, в роботі отримано за допомогою конформного відображення області, 
зайнятої рідиною (півплощини із вирізаним сегментом) на допоміжну півплощину з наступним зведенням вихідної задачі до задачі Келдиша-Ссдова для цісї півплощини.

Дуже істотним с той момент, що застосування принципу Огазо призводить до трансцендентного рівнянню для визначення параметра q, який визначас положення крайньої точки зони відриву, який містить сингулярні інтеграли, які слід розуміти в сенсі кінцевої частини за Адамаром.

Чисельна процедура, заснована на використанні методу Адамара-Манглера, дозволила визначити значення параметра q як функцію кінематичних параметрів i геометричного параметра $\alpha$, що характеризус зазначений сегмент.

Після того як визначено параметр q, який визначає положення зони відриву, визначення потенціалу зводиться до обчислення деякого інтеграла розумісться в сенсі Коші. В роботі представлені результати розрахунку імпульсивного тиску по поверхні сегмента з урахуванням існування ділянки відриву потоку.

Ключові слова: нестисливої рідина, ударна задача для рідини, відрив рідини від твердої поверхні, задача Келдиша-Сєдова

The problem of impact of a smooth curvilinear body previously immersed in a fluid occupying an unlimited half-space in a flat formulation is considered. The fluid is considered incompressible, and the body part immersed in the fluid has the shape of a circular segment. It is assumed that at some point in time, an off-center impact occurs, as a result of which the body instantly receives the horizontal $U$ and vertical $V$ velocity of motion, as well as the angular velocity of rotation $\omega$ around the axis perpendicular to the plane in which the flow is considered.

It is also assumed that with a certain combination of kinematic and geometric parameters of the immersed body part in the form of a segment, the liquid can instantly tear itself away from the body surface and form an additional area of the free surface. The complexity of the problem lies in the fact that the position of the separation zone (the coordinate of its extreme point) is not known in advance; it depends on a combination of kinematic and geometric parameters. The appearance of the separation zone significantly complicates the initial hydrodynamic problem, since the fluid velocity field depends on the position of the separation zone, and the geometric parameters of this zone, in turn, on a combination of kinematic parameters.

In the work, to determine the position of the separation zone (its extreme point), the so-called Ogaso principle was used, which expresses the variational principle, which consists in the fact that the separated flow of liquid realized in reality provides an extreme value of the potential among other possible solutions to the mixed shock hydromechanics problem. This principle allows you to filter out all those possible mathematical solutions that allow the presence of negative impulses on the surface of the contact of the body with the liquid, which contradicts the physical nature of the hydrodynamic phenomena.

The general solution to the problem of determining the field of velocities and momenta in a fluid at the instant immediately following the impact, with a pre-arbitrary parameter characterizing the separation, was obtained by conformally mapping the region occupied by the fluid (half-plane with a cut segment) onto the auxiliary half-plane, and also by reducing the original problem to the Keldysh-Sedov problem for this half-plane.

It is very significant that the application of the Ogaso principle leads to a transcendental equation for determining the parameter $q$, which determines the position of the extreme point of the separation zone, containing singular integrals, which should be understood in the sense of the Adamard finite part.

A numerical procedure based on the Adamard-Mangler method made it possible to determine the value of the parameter $q$ as a function of kinematic parameters and the geometric parameter $\alpha$ characterizing the indicated segment.

After the parameter $q$ is determined, which determines the position of the separation zone, the determination of the potential is reduced to calculating some integral understood in the sense of Cauchy. The paper presents the results of calculating the impulsive pressure over the segment surface, taking into account the existence of a flow separation section.

Keywords: incompressible fluid, shock problem for fluid, separation of fluid from a solid surface, Keldysh-Sedov problem.

Постановка задачи. Задача рассматривается в плоской постановке. Предполагается, что первоначально жидкость покоится и занимает полупространство. В жидкость частично погружено цилиндрическое тело, поперечное сечение погруженной части которого имеет криволинейную форму в 
виде кругового сегмента; ширина сегмента, измеренная вдоль свободной поверхности жидкости (хорда сегмента), равна $2 a$; угол сегмента (угол между касательной к образующей в точке контакта поверхности сегмента со свободной поверхностью и хордой сегмента) обозначим $\alpha$.

Расположим декартову систему координат Oxy так, чтобы ось у была направлена вертикально вглубь жидкости, а вдоль ее свободной поверхности направим ось х. Начало координат совместим с точкой пересечения оси симметрии погруженной части тела с осью $x$. Таким образом, жидкость находится в верхней полуплоскости у $\geq 0$ системы координат $O x y$ (рис. 1).

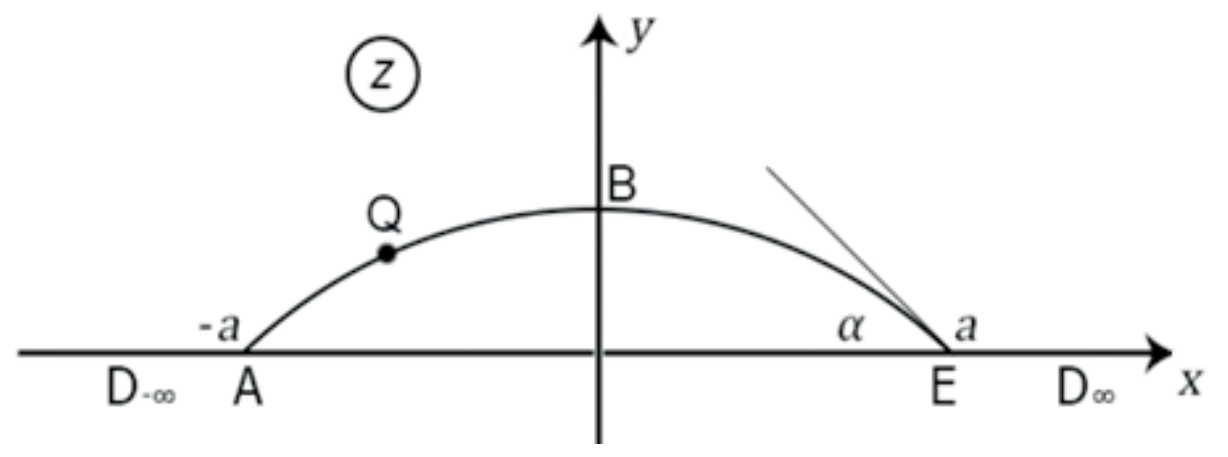

Рис. 1. Схема к постановке задачи

Предполагается, что в некоторый начальный момент времени происходит нецентральный удар, после чего тело мгновенно получает вертикальную скорость $V$, горизонтальную скорость $U$ и вращательную скорость $\omega$ вокруг оси, перпендикулярной плоскости, в которой рассматривается течение жидкости.

В результате удара возникнет потенциальное течение жидкости, которое будем описывать комплексным потенциалом:

$$
w(z)=\varphi(x, y)+i \psi(x, y)
$$

Здесь $\mathrm{z}=\mathrm{x}+\mathrm{iy}-$ комплексная переменная, $\varphi(\mathrm{x}, \mathrm{y})-$ потенциал скорости, $\psi(\mathrm{x}, \mathrm{y})-$ функция тока.

В момент непосредственно после удара жидкость может начать двигаться с образованием зоны отрыва, положение которой заранее неизвестно и подлежит определению. Зона отрыва может возникнуть на том участке предварительного контакта поверхности тела с жидкостью, на котором нормальная компонента скорости точек поверхности тела $\mathrm{V}_{\mathrm{Tn}}$ направлена вне жидкости, и в результате жидкость, в силу своей инерции, может не успеть за движением поверхности тела, и тогда между поверхностью тела и жидкостью мгновенно образуется бесконечно тонкая щель и начинает формироваться новый участок свободной поверхности жидкости. Как показывает опыт, новый участок свободной поверхности, как правило, примыкает к уже существующему участку свободной поверхности.

До момента удара в рассматриваемой постановке задачи имеется два прямолинейных участка свободной границы: $\mathrm{D}_{-\infty} \mathrm{A}$ и $\mathrm{ED}_{\infty}$. Будем предполагать, что начальные условия удара (то есть компоненты скорости U, V и угловая скорость $\omega$ ) таковы, что зона отрыва примыкает к участку свободной поверхности $\mathrm{D}_{-\infty} \mathrm{A}$ и представляет собой дугу окружности $\mathrm{AO}$, положение крайней точки которой $\mathrm{Q}$ 
заранее неизвестно и представляет собой главный предмет поиска рассматриваемой задачи. Остальная часть поверхности тела QBE после удара продолжает оставаться в контакте с жидкостью и на этой части соблюдается обычное условие безотрывного обтекания.

Задачу будем решать в терминах $\varphi$ и $\psi$. Поэтому и граничные условия будем использовать в тех же терминах. Как известно [3], при ударном взаимодействии на жидкость в ней возникает потенциальное течение с потенциалом $\varphi$, который связан с импульсным давлением $\mathrm{P}_{\mathrm{t}}$ зависимостью:

$$
\mathrm{P}_{\mathrm{t}}=-\rho \varphi
$$

Поскольку на свободных поверхностях жидкости (в том числе и на вновь образовавшейся поверхности отрыва $\mathrm{AQ}$ ) импульс $\mathrm{P}_{\mathrm{t}}$ отсутствует, то для решения поставленной задачи будем иметь следующие граничные условия: на участках свободной границы $\mathrm{D}_{-\infty} \mathrm{A}$ и $\mathrm{ED}_{\infty}$, а также на том участке тела $\mathrm{AQ}$, на котором произошел отрыв, имеем:

$$
\varphi=0 \text {. }
$$

На смоченной поверхности тела QBE имеем условие безотрывного обтекания, которое через функцию тока выражается в виде [4]:

$$
\left.\psi\right|_{\mathrm{QBE}}=U \mathrm{U}-\mathrm{Vx}-\frac{\omega}{2}\left(\mathrm{x}^{2}+\mathrm{y}^{2}\right) \text {. }
$$

Таким образом, для решения гидродинамической задачи об определении мгновенного поля скоростей в жидкости, занимающую безграничную область, поперечное сечение которой представляет полуплоскость с вырезанной лункой, имеем смешанную задачу теории аналитических функций.

Отметим, что ранее в нашей работе [1] задача об ударном взаимодействии криволинейной поверхности в виде кругового сегмента уже рассматривалась в предположении об отсутствии отрыва жидкости от твердой поверхности тела. Оказалось, что математическая задача имеет решение при любой комбинации начальных кинематических параметров U, V и $\omega$. Однако при некоторых их комбинациях на поверхности тела возникают положительные значения потенциала (то есть, отрицательные значения импульса давления), что противоречит физической сущности и свидетельствует о необходимости перестройки физики явления и, следовательно, постановки математической задачи с учетом явления отрыва.

Решение задачи. Для решения полученной задачи удобно ввести характеристическую функцию

$$
\chi=-\mathrm{iw}=\psi-\mathrm{i} \varphi,
$$

и свести полученную смешанную задачу к классической краевой задаче КелдышаСедова со смешанными граничными условиями для полуплоскости, [2, 6, 7]. Совершим конформное отображение верхней полуплоскости с указанной лункой в физической плоскости $\mathrm{z}=\mathrm{x}+$ iу на верхнюю полуплоскость вспомогательной комплексной переменной $\zeta=\xi+$ i . При этом соблюдается соответствие: точка $\mathrm{z}=$ а переходит в точку $\zeta=1$, точка $\mathrm{z}=-$ а переходит в $\zeta=-1$, а точка $\mathrm{z}=\infty-$ в $\zeta=\infty$ 
(точка $\mathrm{z}=\mathrm{B}$, по симметрии, переходит в $\zeta=0$ ), и пусть точке $\mathrm{Q}$ соответствует $\xi_{\mathrm{Q}}=$ $-q$ (рис. 2).

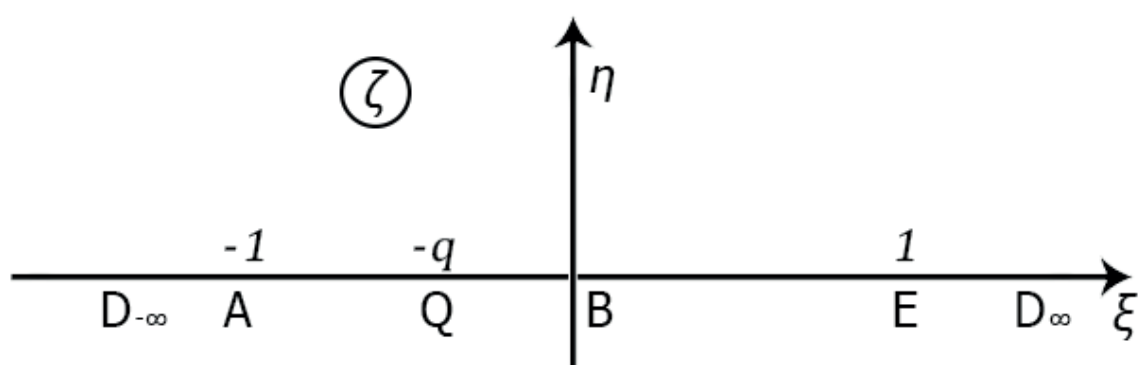

\section{Рис. 2. Область параметрической переменной}

Указанное конформное отображение имеет вид, [5]:

$$
\zeta(z)=\frac{(z+a)^{\frac{\pi}{\pi-\alpha}}+(z-a)^{\frac{\pi}{\pi-\alpha}}}{(z+a)^{\frac{\pi}{\pi-\alpha}}-(z-a)^{\frac{\pi}{\pi-\alpha}}} .
$$

Для обратного преобразования имеем зависимость:

$$
z(\zeta)=a \frac{(\zeta+1)^{\frac{\pi-\alpha}{\pi}}+(\zeta-1)^{\frac{\pi-\alpha}{\pi}}}{(\zeta+1)^{\frac{\pi-\alpha}{\pi}}-(\zeta-1)^{\frac{\pi-\alpha}{\pi}}}
$$

В новых переменных задача Келдыша-Седова для верхней полуплоскости имеет следующую постановку: определить функцию $\chi(z(\zeta))$ при $\operatorname{Im} \zeta>0$ по следующим граничным условиям: на образе свободной поверхности и зоны отрыва $(-\infty<\xi<$ $-q$ и $1<\xi<\infty): \varphi=0$; на образе участка безотрывного обтекания поверхности тела $(-q<\xi<1): \psi(z(\zeta))=\psi(\xi)$. Функция $\psi(\zeta)$ на смоченной поверхности тела в этом случае примет следующий вид:

$$
\psi(\xi)=U \psi_{1}(\xi)+V \psi_{2}(\xi)+\frac{\omega \mathrm{a}}{2} \Psi_{3}(\xi)
$$

где

$$
\begin{gathered}
\psi_{1}(\xi)=\frac{\phi_{1}(\xi)}{\phi_{0}(\xi)} ; \quad \Psi_{2}(\xi)=\frac{\phi_{2}(\xi)}{\phi_{0}(\xi)} ; \quad \Psi_{3}(\xi)=\frac{\phi_{1}^{2}(\xi)+\phi_{2}^{2}(\xi)}{\phi_{0}^{2}(\xi)} ; \\
\phi_{1}(\xi)=2 \mathrm{~F}(\xi) \sin \alpha ; \quad \phi_{2}(\xi)=\mathrm{F}^{2}(\xi)-1 ; \quad \phi_{0}(\xi)=\mathrm{F}^{2}(\xi)+2 \mathrm{~F}(\xi) \cos \alpha+1 ; \\
\mathrm{F}(\xi)=\left(\frac{1+\xi}{1-\xi}\right)^{\frac{\pi-\alpha}{\pi}} .
\end{gathered}
$$

Теперь можем записать формулу Келдыша-Седова для решения поставленной задачи в верхней полуплоскости в новых переменных, [6]: 


$$
\chi(\zeta)=-\frac{1}{\pi} \sqrt{(\zeta-1)(\zeta+q)} \int_{-q}^{1} \frac{\psi(t)}{\sqrt{(1-t)(t+q)}} \frac{d t}{t-\zeta} .
$$

Чтобы конструктивно воспользоваться это формулой, нужно знать числовое значение параметра q. Однако этот параметр, представляющий координату крайней точки зоны отрыва на вспомогательной плоскости $\zeta$, остается заранее неизвестным. Для его определения необходимо некоторое дополнительное условие, которое могло бы реализоваться в уравнение для вычисления величины q. Обычно в качестве этого условия использовался критерий Седова [7] о равенстве нормальных компонент скорости в предельной точке области отрыва со стороны отрыва и со стороны безотрывного течения.

Однако в последнее время стал применяться принцип Огазо [8], который в применении к нашей задаче может быть выражен в виде следующего предельного равенства:

$$
\begin{aligned}
& \lim _{\xi \rightarrow-q+0} \frac{\partial \varphi}{\partial q} \\
& =0
\end{aligned}
$$

где $\varphi(\xi, q)$ - значение потенциала на гладком участке безотрывного обтекания как функция от $\xi$.

Уравнение Огазо выражает вариационный принцип следующего содержания: реализуемое в действительности отрывное течение обеспечивает экстремальное значение потенциала среди других возможных решений смешанной ударной задачи гидромеханики. Уравнение (3) служит для определения параметра q, если известно общее решение задачи $\varphi(\xi, \mathrm{q})$ для произвольного значения q.

Таким образом, чтобы воспользоваться условием Огазо, нужно уметь находить решение краевой задачи при произвольных предполагаемых значениях q, а затем из условия (3) определить то значение q, которое и будет, по предположению, определять положение точки отрыва.

Принцип Огазо позволяет отсеять все те решения, которые допускают возникновение на поверхности контакта тела с жидкостью отрицательных импульсов. Поскольку по физической сущности, импульс может быть только положительным (или, в крайнем случае, нулевым), такая возможность обеспечивается тем, что на поверхности тела в определенном месте должен возникать отрыв.

Итак, для возможности использования принципа Огазо, необходимо иметь выражение для $\varphi(\xi, q)$ на поверхности тела, зависящее от q как от параметра. Эта функция определяется из выражения (1) при помощи формулы Сохоцкого и имеет вид:

$$
\begin{aligned}
& \varphi(\xi, q) \\
& =\frac{1}{\pi} \sqrt{(1-\xi)(\xi+q)} \int_{-q}^{1} \frac{\psi(t)}{\sqrt{(1-t)(t+q)}} \frac{d t}{t-\xi},
\end{aligned}
$$

где интеграл понимается в смысле главного значения по Коши. 
В соответствии со структурой функции $\psi(\xi)(1)$, для функции $\varphi(\xi, q)$ получаем выражение вида:

$$
\begin{gathered}
\varphi(\xi, \mathrm{q})=\mathrm{U} \varphi_{1}(\xi, \mathrm{q})+\mathrm{V} \varphi_{2}(\xi, \mathrm{q})+\frac{\omega \mathrm{a}}{2} \varphi_{3}(\xi, \mathrm{q}) \\
\varphi_{\mathrm{k}}(\xi, \mathrm{q})=\frac{1}{\pi} \sqrt{(1-\xi)(\xi+\mathrm{q})} \int_{-\mathrm{q}}^{1} \frac{\psi_{\mathrm{k}}(\mathrm{t})}{\sqrt{(1-\mathrm{t})(\mathrm{t}+\mathrm{q})}} \frac{\mathrm{dt}}{\mathrm{t}-\xi}
\end{gathered}
$$

Вычисляя производную от функции $\varphi(\xi, q)(5)$ по параметру q и переходя к пределу согласно критерию Огазо (3), получим следующее трансцендентное уравнение для определения параметра q:

$$
\mathrm{UI}_{1}(-\mathrm{q})+\mathrm{VI}_{2}(-\mathrm{q})+\frac{\omega \mathrm{a}}{2} \mathrm{I}_{3}(-\mathrm{q})=0
$$

где введены обозначения

$$
I_{k}(-q)=\int_{-q}^{1} \frac{\psi_{k}(\xi) d \xi}{(\xi+q)^{\frac{3}{2}}(1-\xi)^{\frac{1}{2}}}, k=1,2,3
$$

Все интегралы на пределе $\xi=-q$ имеют подынтегральную сингулярную особенность порядка $(\xi+q)^{-3 / 2}$, интегрируемую в смысле Адамара-Манглера.

Уравнение (6) за счет введения двух кинематических параметров

$$
\frac{\mathrm{U}}{\mathrm{V}}=\operatorname{tg} \beta \text { и } \mathrm{St}=\frac{\omega \mathrm{a}}{\mathrm{V}}
$$

(параметр St играет роль числа Струхаля) может быть сведено к виду:

$$
\mathrm{I}_{2}(-\mathrm{q})+\frac{\mathrm{U}}{\mathrm{V}} \mathrm{I}_{1}(-\mathrm{q})+\frac{1}{2} \mathrm{StI}_{3}(-\mathrm{q})=0
$$

Из выражения (8) видно, что искомый параметр q является функцией двух кинематических параметров: угла наклона вектора индуцированной скорости $\beta=$ $\operatorname{arctg} \frac{\mathrm{U}}{\mathrm{V}}$ и числа Струхаля $\mathrm{St}=\frac{\omega \mathrm{a}}{\mathrm{V}}$, а также геометрического параметра $\alpha$, характеризующего выпуклость кругового сегмента погруженной части тела.

Для численного решения уравнения (8) применялась основная формула Адамара-Манглера

$$
\int_{a}^{x} \frac{A(y) d y}{(x-y)^{3 / 2}}=\int_{a}^{x} \frac{A(y)-A(x)}{(x-y)^{3 / 2}} d y-A(x) \frac{2}{\sqrt{x-a}}
$$

где интеграл справа, в силу предположенной дифференцируемости функции $\mathrm{A}(\mathrm{y})$, в точке $\mathrm{y}=\mathrm{x}$ имеет интегрируемую особенность порядка $(\mathrm{x}-\mathrm{y})^{-1 / 2}$. При практическом вычислении интегралы $\mathrm{I}_{\mathrm{k}}(-\mathrm{q})$ предварительно приводились к стандартному виду при помощи замены $\xi=-\mathrm{y}$ : 
где

$$
\mathrm{I}_{\mathrm{k}}(-\mathrm{q})=\int_{-\mathrm{q}}^{1} \frac{\psi_{\mathrm{k}}(\xi) \mathrm{d} \xi}{(\xi+\mathrm{q})^{\frac{3}{2}}(1-\xi)^{\frac{1}{2}}}=\int_{-1}^{\mathrm{q}} \frac{\mathrm{A}_{\mathrm{k}}(\mathrm{y}) \mathrm{dy}}{(\mathrm{q}-\mathrm{y})^{\frac{3}{2}}}
$$

$$
A_{k}(y)=\frac{\psi_{k}(-y)}{(1+y)^{\frac{1}{2}}}
$$

Таким образом, стандартная процедура Адамара-Манглера для вычисления каждого из интегралов $\mathrm{I}_{\mathrm{k}}$ имела следующий вид:

$$
\int_{-1}^{\mathrm{q}} \frac{A_{k}(y) d y}{(q-y)^{\frac{3}{2}}}=\int_{-1}^{q} \frac{A_{k}(y)-A_{k}(q) d y}{(q-y)^{\frac{3}{2}}}-2 \frac{\psi_{k}(-q)}{1+q}
$$

Анализ результатов. В результате, была решена плоская задача КелдышаСедова для тела, имеющего форму кругового сектора. Имея выражение для нахождения точки отрыва жидкости от поверхности тела при ударе, сделаем анализ полученных результатов.

Как следует из выражений, полученных выше и из результатов решения уравнения (8) при помощи применения формулы Адамара-Манглера, положение точки отрыва зависит от отношения компонентов скорости и угла сегмента $\alpha$ нелинейным образом. Проиллюстрируем эту зависимость при разных $\alpha$, $\omega$ и отношениях поступательных скоростей $U / V$. На рис. 3-6 координата точки отрыва приведена во вспомогательной плоскости $\zeta$.

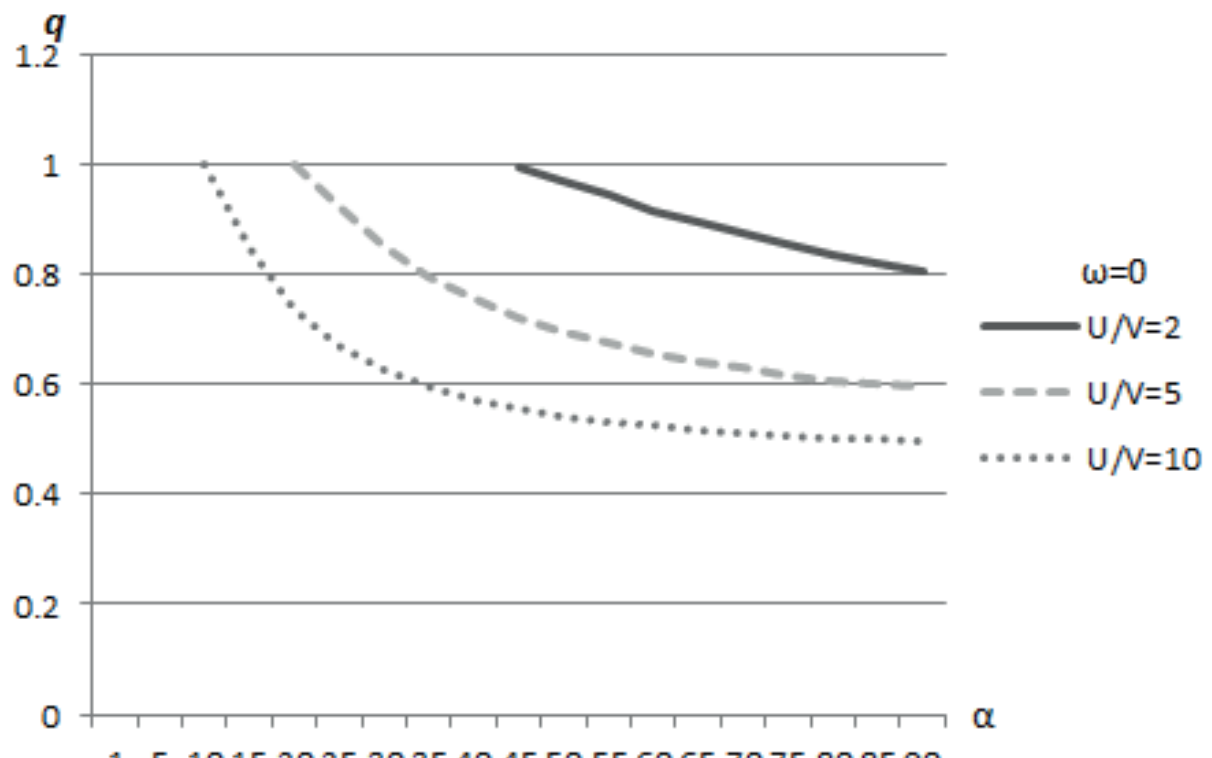

151015202530354045505560657075808590

Рис. 4. Зависимость положения точки отрыва от угла сегмента и отношения поступательных скоростей, при отсутствии вращения 


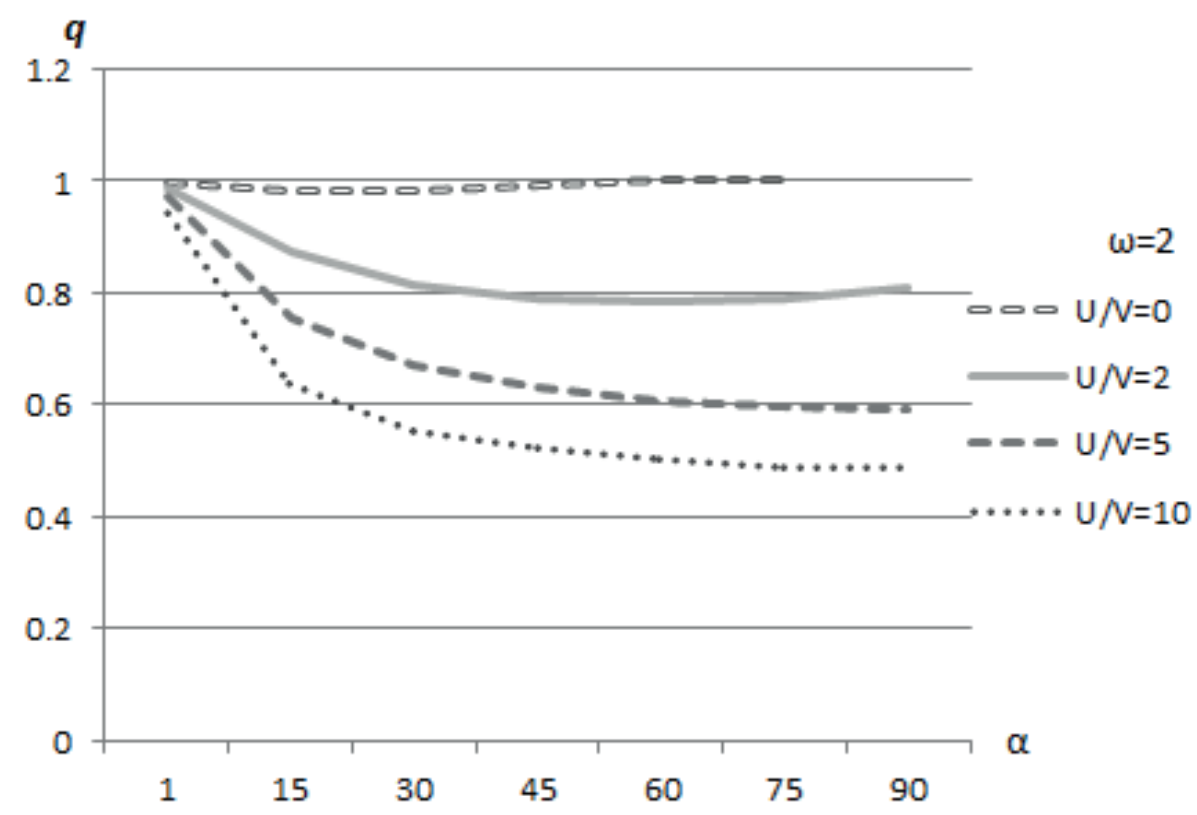

Рис. 5. Зависимость положения точки отрыва от угла сегмента и отношения поступательных скоростей, при $\omega=2$

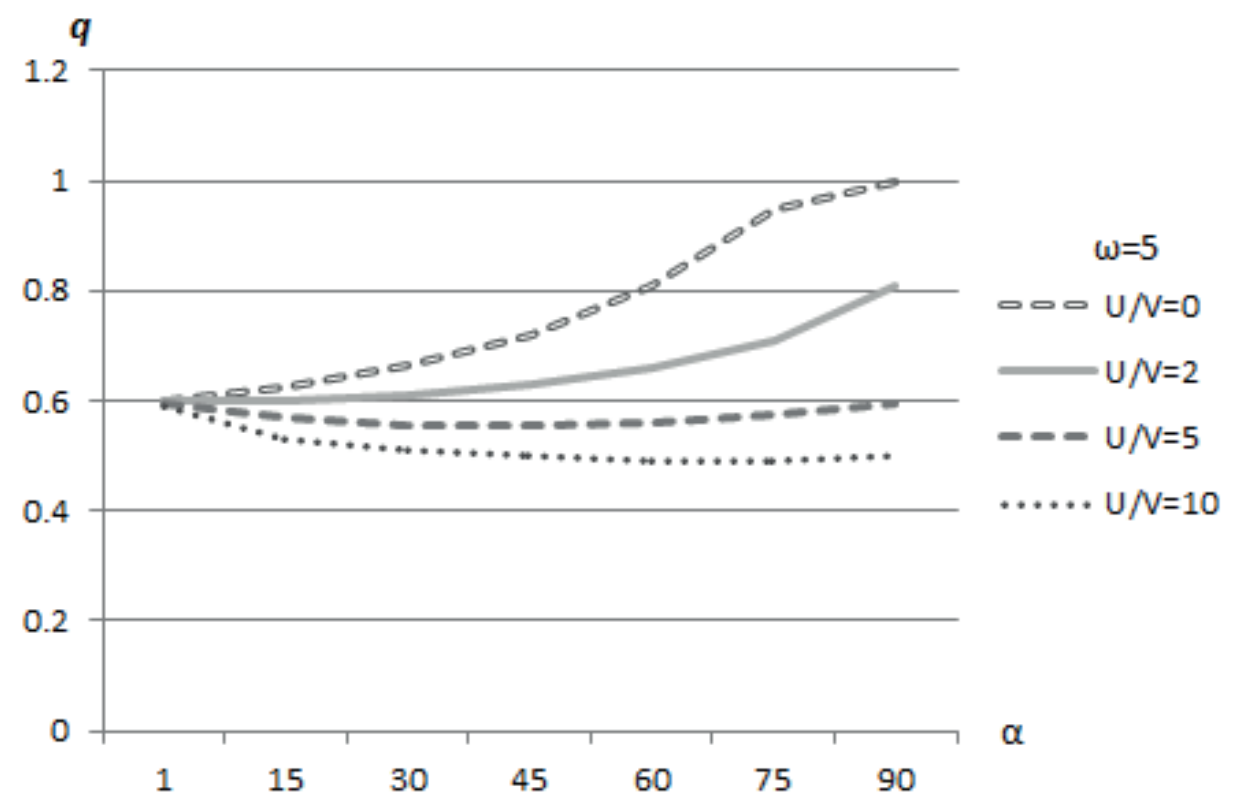

Рис. 6. Зависимость положения точки отрыва от угла сегмента и отношения поступательных скоростей, при $\omega=5$ 


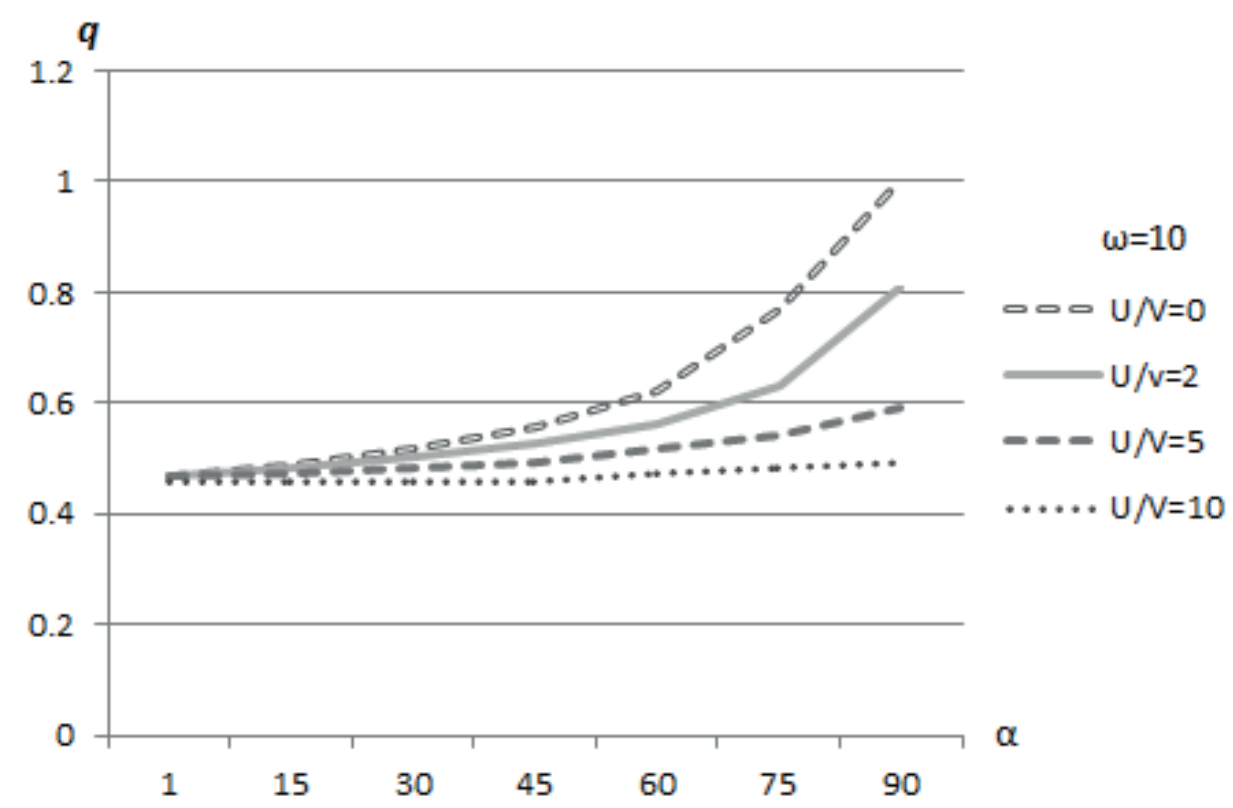

Рис. 7. Зависимость положения точки отрыва от угла сегмента и отношения поступательных скоростей, при $\omega=10$

Проиллюстрируем эпюры импульсного давления жидкости на тело при разных параметрах скорости и угла сегмента. Здесь жидкость находится в верхней полуплоскости.

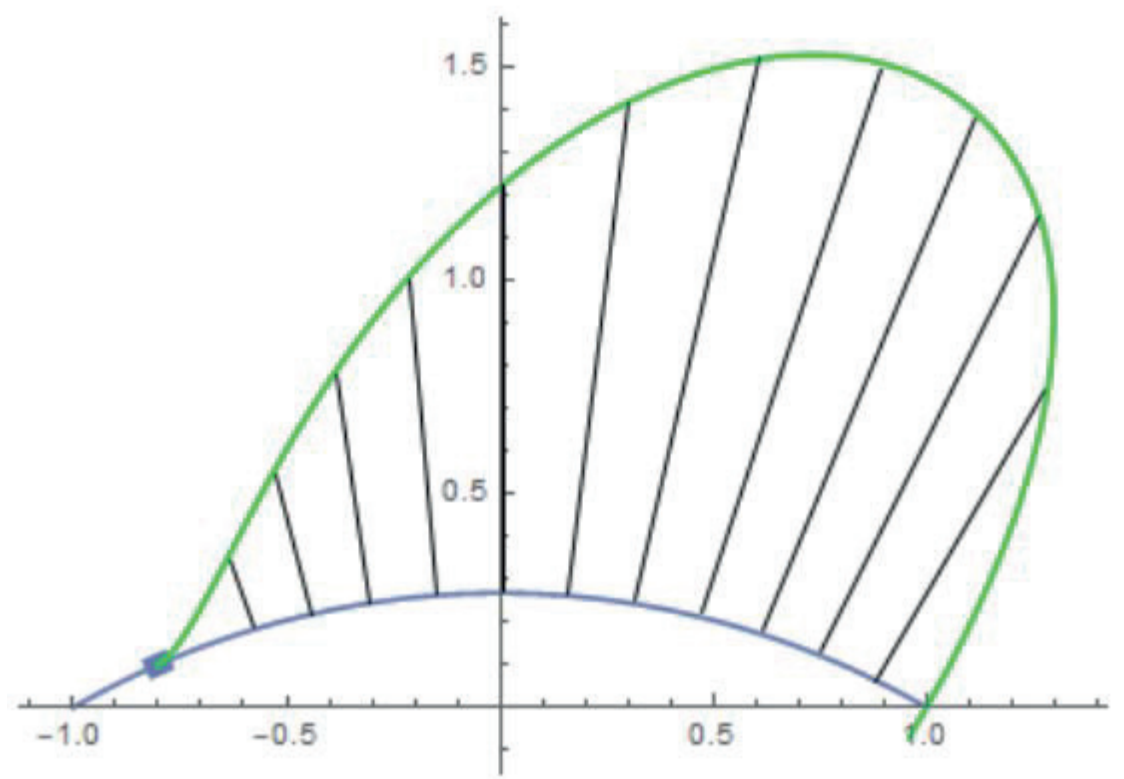

Рис. 8. Эпюра импульсного давления жидкости на тело: $U / V=5, \omega=0, \alpha=30$ 


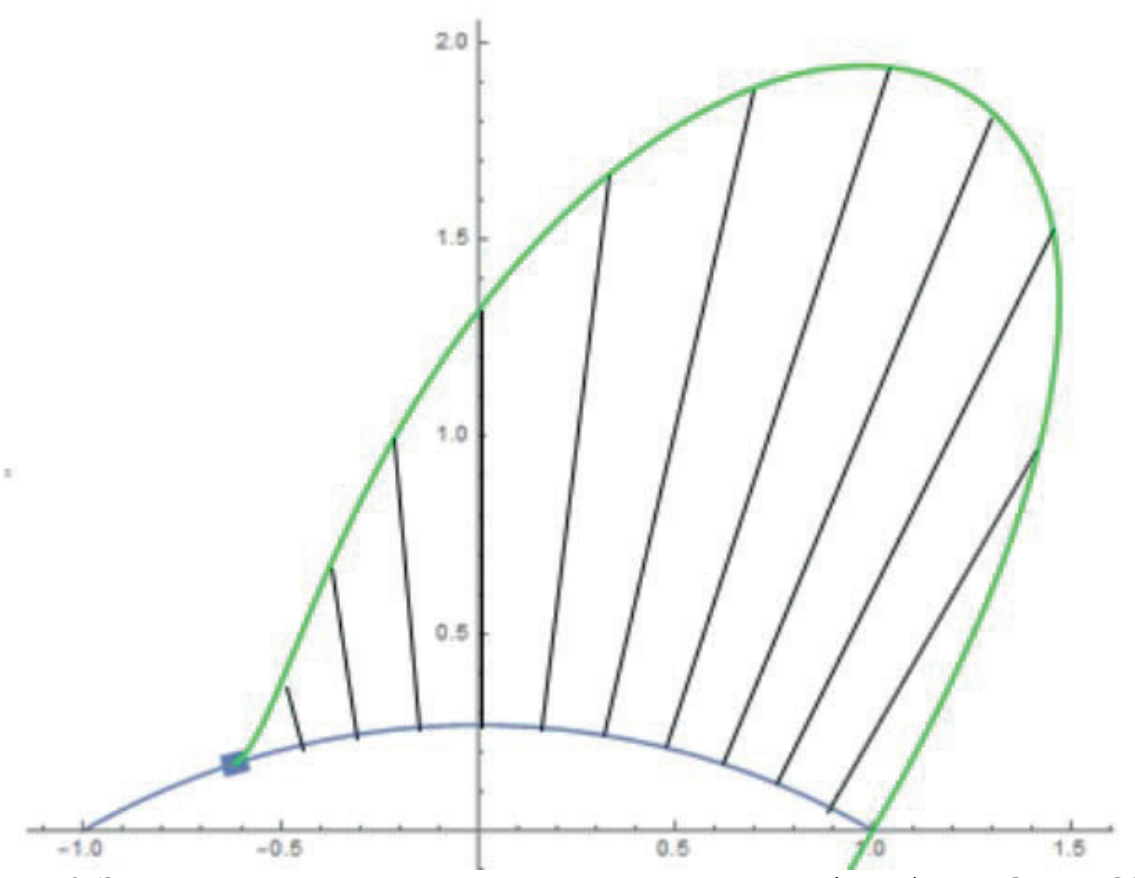

Рис. 9. Эпюра импульсного давления жидкости на тело: $U / V=5, \omega=2, \alpha=30$

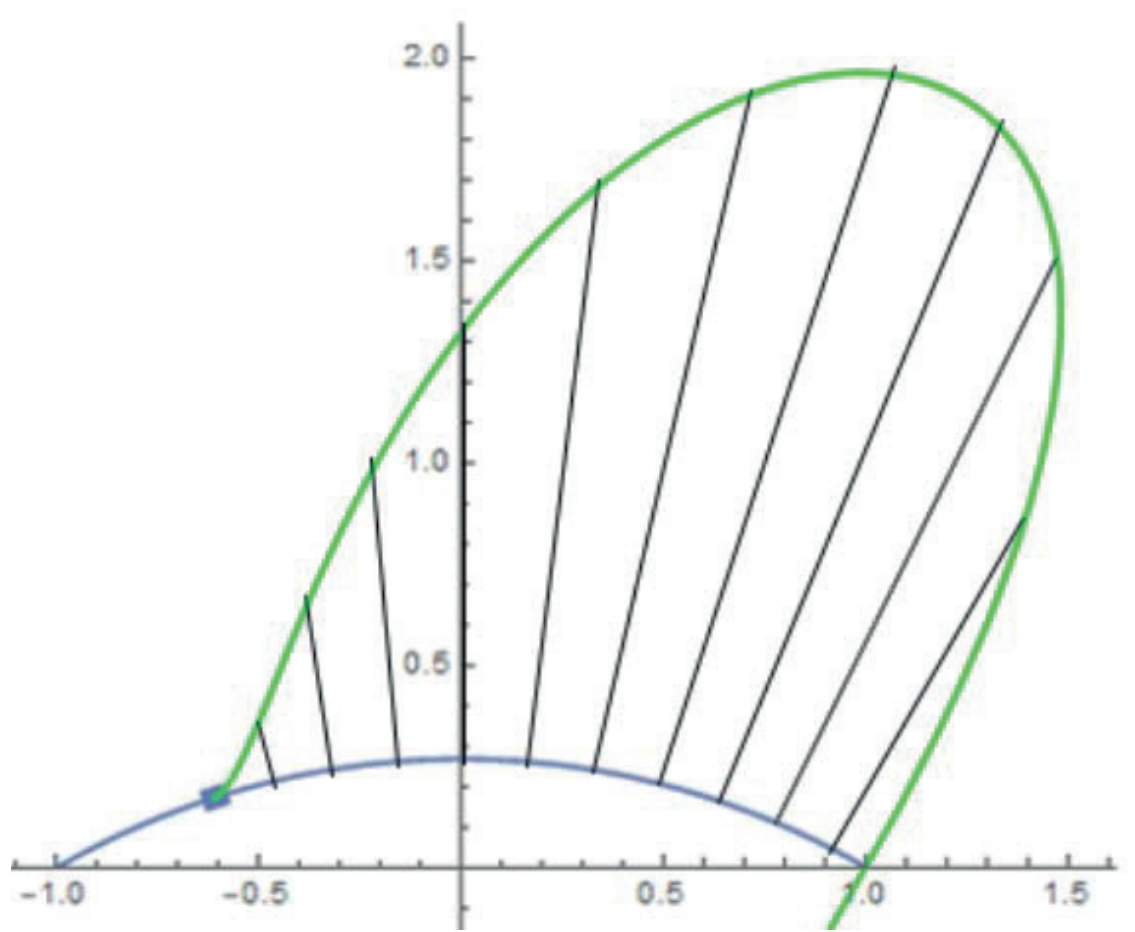

Рис. 10. Эпюра импульсного давления жидкости на тело: $\mathrm{U} / \mathrm{V}=0, \omega=5, \alpha=30$

\section{БИБЛИОГРАФИЧЕСКИЕ ССЫЛКИ}

1. Гоман, О. Г. Удар круглого тела о поверхность идеальной несжимаемой жидкости [Текст] / О. Г. Гоман, Т. М. Никулина // // Вісн. Дніпр. ун-ту. Сер.: Механіка. - 2018. - Вип. 5, т. $26 .-$ C. $50-57$ 
2. Гахов, Ф. Д. Краевые задачи [Текст] / Ф. Д. Гахов. - М.: Наука, 1977. - 640 с.

3. Гоман, О. Г. Ударное взаимодействие несжимаемой жидкости и вертикальной пластины, плавающей на ее поверхности, в условиях образования одной зоны отрыва и налички вращения [Текст] / О. Г. Гоман, В. А. Катан // Вісн. Дніпр. ун-ту. Сер.: Механіка. 2013. - Вип. 17, т. 1. - С. 191-205.

4. Кочин, Н. Е. Теоретическая гидромеханика в 2-х ч. [Текст] / Н. Е. Кочин, И. А. Кибель, Н. В. Розе. - М.: Гостехиздат, ч. 1. - 1948. - 536 с.

5. Лаврентьев, М. А. Методы теории функций комплексного переменного [Текст] / М. А. Лаврентьев, Б. В. Шабат. - М.: Наука, 1973. - 736 с.

6. Мусхелишвилли, Н. И. Сингулярные интегральные уравнения [Текст] / Н. И. Мусхелишвилли. - М.: Наука, 1968. - 512 с.

7. Седов, Л. И. Плоские задачи гидродинамики и аэродинамики [Текст] / Л. И. Седов. - М.: Наука, 1966. - 448 с.

8. Норкин, М. В. Смешанные задачи гидродинамического удара / М. В. Норкин. Ростов-на-Дону, 2007. - 136 с.

\title{
МЕТОДИКА РАСЧЕТА ВЕЛИЧИНЫ ПРОВАЛА ДАВЛЕНИЯ НА ВХОДЕ В ЭЛЕКТРОНАСОСЫ ПРИ ЗАПРАВКЕ РАКЕТОНОСИТЕЛЯ С АНАЛИЗОМ ПОЛУЧЕННЫХ ЭКСПЕРИМЕНТАЛЬНЫХ ДАННЫХ
}

\begin{abstract}
Целью исследований было определение схемы обвязки и режимов работы насосов системы заправки компонентами ракетного топлива (КРТ) баков ракетоносителя для обеспечения требований по их эксплуатации. Задачей было определение влияния параметров КРТ и гидравлической системы на величину провала давления на входе в насос. Под провалом давления понимается разница между стационарным давлением (после выхода на режим) и минимальным давлением на входе в насос. Кавитация и разнасыщение рабочей жидкости на входе в насос возможны при падении давления ниже давления насыщенных паров. Кавитация - это быстротекущие физико-механические процессы в жидкости, возникающие при уменьшении давления до величины, менышей чем давление насыщенных паров при данной температуре. При этом в отдельных местах потока возникает разрыв сплошности с заполнением пустот мелкими пузырьками насыщенного пара и выделившегося из жидкости растворенного воздуха. Кавитация, как правило, приводит к снижению напора, подачи и КПД насоса, возникновению шума и вибраций. У центробежных насосов зона возникновения кавитационных явлений находится вблизи входа в рабочее колесо. Разработана методика расчета величины провала давления на входе в насос в режиме включения при заправке РН, подтвержденная экспериментально. Включение насоса происходило при закрытом клапане на напорном трубопроводе, который открывается после выхода насоса на номинальный режим. В зависимости от времени открытия клапана меняется ускорение потока жидкости в трубопроводе. Представлены экспериментальные данные по амплитуде и длительности провала давления в зависимости от параметров КРТ и гидравлической системы.
\end{abstract}

(с) Кузьмич И. Ю., Г., Седых И. В., Минай А. В., 2019 